\title{
Diazoxide amelioration of myocardial injury and mitochondrial damage during cardiac surgery
}

\section{Citation}

McCully, James D, Hidetaka Wakiyama, Douglas B Cowan, Micheline Federman, Robert A Parker, and Sidney Levitsky. 2002. "Diazoxide Amelioration of Myocardial Injury and Mitochondrial Damage During Cardiac Surgery." The Annals of Thoracic Surgery 74 (6) (December): 2138-2146. doi:10.1016/s0003-4975(02)04348-5.

\section{Published Version}

doi:http://dx.doi.org/10.1016/S0003-4975(02)04348-5

\section{Permanent link}

http://nrs.harvard.edu/urn-3:HUL.InstRepos:27363790

\section{Terms of Use}

This article was downloaded from Harvard University's DASH repository, and is made available under the terms and conditions applicable to Other Posted Material, as set forth at http:// nrs.harvard.edu/urn-3:HUL.InstRepos:dash.current.terms-of-use\#LAA

\section{Share Your Story}

The Harvard community has made this article openly available.

Please share how this access benefits you. Submit a story.

\section{Accessibility}




\title{
Diazoxide Amelioration of Myocardial Injury and Mitochondrial Damage During Cardiac Surgery
}

\author{
James D. McCully, PhD, Hidetaka Wakiyama, MD, Douglas B. Cowan, PhD, Micheline \\ Federman, PhD, Robert A. Parker, ScD, and Sidney Levitsky, MD \\ Divisions of Cardiothoracic Surgery and Pathology and Biometrics Center, Beth Israel Deaconess \\ Medical Center, Department of Anesthesiology, Children's Hospital, and Harvard Medical School, \\ Boston, Massachusetts
}

\section{Abstract}

Background-Recently, we have shown that the selective opening of mitochondrial ATPsensitive potassium channels with diazoxide significantly decreases myocardial injury. The purpose of this study was to determine the effects of diazoxide on apoptosis and the mechanisms modulating apoptosis and myocardial injury in a blood-perfused model of acute myocardial infarction.

Methods-Pigs ( 32 to $42 \mathrm{~kg}$ ) undergoing total cardiopulmonary bypass underwent left anterior descending coronary artery occlusion for 30 minutes. The aorta was cross-clamped and magnesium-supplemented potassium cold-blood cardioplegia (DSA; $n=6)$ or magnesiumsupplemented potassium cardioplegia containing $50 \mu \mathrm{mol} / \mathrm{L}$ diazoxide $(\mathrm{DZX} ; \mathrm{n}=6$ ) was administered, followed by 30 minutes of global ischemia and 120 minutes of reperfusion. Left ventricular tissue samples from DSA and DZX hearts were obtained after reperfusion. Apoptosis was determined by TUNEL, caspase- 3 and PARP cleavage, and caspase- 3 activity. Bax and bcl- 2 levels were determined and tissue morphology was examined by light and transmission electron microscopy.

Results-Apoptosis, as estimated by TUNEL-positive nuclei/3,000 myocardial cells, was 120.3 \pm 48.8 in DSA hearts and was significantly decreased to $21.4 \pm 5.3$ in DZX hearts $(p<0.05$ vs control). Caspase- 3 and poly-ADP-ribose polymerase cleavage and pro-apoptotic bax protein levels were significantly decreased with diazoxide ( $p<0.05$ vs DSA). Light and transmission electron microscopy indicated severe disruption of tissue with capillary dilatation, mitochondrial cristae damage, and evidence of increased presence of mitochondrial granules in DSA as compared with DZX hearts.

Conclusions-The addition of diazoxide ( $50 \mu \mathrm{mol} / \mathrm{L}$ ) to cardioplegia significantly decreases regional myocardial apoptosis and mitochondrial damage, and provides an additional modality for achieving myocardial protection.

Acute myocardial infarction is the leading cause of morbidity and mortality in the US. Cardiac surgery in the management of these high-risk patients can provide an integral component in the early-phase management of acute myocardial infarction, but effective cardioplegia formulations that would allow for enhanced tissue preservation in this high-risk group have yet to be determined.

(C) 2002 by The Society of Thoracic Surgeons

Address reprint requests to Dr McCully, Division of Cardiothoracic Surgery, Beth Israel Deaconess Medical Center, Harvard Institutes of Medicine, 77 Ave Louis Pasteur, Room 144, Boston MA 02115; james_mccully@hms.harvard.edu..

Presented at the Thirty-eighth Annual Meeting of The Society of Thoracic Surgeons, Fort Lauderdale, FL, Jan 28-30, 2002. 
Previously, we have shown that magnesium-supplemented potassium cardioplegia (Deaconess Surgical Associates [DSA], Boston, MA) provides enhanced cardioprotection as compared with traditional high-potassium cardioplegia. The mechanisms by which DSA cardioplegia affords enhanced cardioprotection involve the amelioration of cytosolic, mitochondrial, and nuclear calcium overload, enhanced preservation and resynthesis of high-energy phosphates, and the modulation of nuclear and mitochondrial function [1-3]. The end effector of these mechanisms remains to be elucidated; however, recent investigations have suggested that the mitochondrial ATP-sensitive potassium (mitoK $\mathrm{ATP}_{\mathrm{AT}}$ ) channels play a predominant role in the infarct-limiting effects afforded by DSA cardioplegia $[4,5]$.

Previous investigations in isolated crystalloid-perfused, in situ blood-perfused animal models and in vitro studies using human trabeculae have demonstrated that nonspecific potassium channel openers such as nicorandil and pinacidil, which allow for unmodulated opening of both sarc- and mitoK $\mathrm{K}_{\mathrm{ATP}}$ channels, significantly enhance cardioprotection when used alone. However, these cardioprotective effects, when used in conjunction with cardioplegia, are limited or inhibited altogether [6-8].

Recently, we have investigated the cardioprotective effects of diazoxide, a cardioselective mitoK $_{\text {ATP }}$ channel opener in conjunction with DSA cardioplegia, using a clinically relevant model of acute myocardial infarction in the in situ blood-perfused pig heart [4]. Our results indicated that the pharmacological opening of mitoK $\mathrm{ATP}_{\mathrm{AT}}$ channels with diazoxide $(50 \mu \mathrm{mol} /$ L) with DSA cardioplegia significantly decreases infarct size ( $p<0.05$ vs DSA) as compared with DSA cardioplegia alone, and that these enhanced infarct-limiting effects are independent of vasodilatation [4].

At least two morphologically distinct pathways, necrosis and apoptosis, have been shown to contribute to myocardial infarct size after ischemia/reperfusion [9]. In this paper, we investigate the effects of the specific mitoK $_{\mathrm{ATP}}$ channel opener, diazoxide, when added to DSA cardioplegia on the modulation of apoptosis after ischemia/reperfusion, and the mechanisms modulating apoptosis in an in situ blood-perfused model of acute myocardial infarction.

\section{Material and Methods}

\section{Animals}

Animals were housed individually and provided with laboratory chow and water ab libitum. All experiments were approved by the Beth Israel Deaconess Medical Center Animal Care and Use Committee and the Harvard Medical Area Standing Committee on Animals (Institutional Animal Care and Use Committee) and conformed to the National Institutes of Health guidelines regulating the care and use of laboratory animals (National Institutes of Health publication 5377-83, 1996).

\section{Surgical Preparation}

Yorkshire pigs of either gender ( 32 to $42 \mathrm{~kg}, \mathrm{n}=15$ ) were sedated with ketamine hydrochloride (20 mg/kg, IM; Abbott Laboratories, North Chicago, IL), and anesthetized with a bolus infusion of thiopental sodium $(5.0$ to $7.0 \mathrm{mg} / \mathrm{kg}$ IV; Baxter Healthcare Corporation, Inc, Deerfield, IL) through an ear vein. Surgical preparation (sternotomy, tracheotomy, cannulation) was performed as previously described [4]. General anesthesia was maintained with 3.0\% sevoflurane (Ultane; Abbott Laboratories, Inc) at the beginning of the surgical preparation, and then reduced to $1.0 \%$ and maintained at this concentration throughout the experiment. During cardiopulmonary bypass (CPB), propofol (0.5 to $0.7 \mathrm{mg} /$ $\mathrm{kg} / \mathrm{min}$ IV; Baxter Healthcare Corporation, Inc) was continuously infused through the 
jugular vein. Heparin sodium (5,000 IU IV; Elkins-Sinn, Inc, Cherry Hill, NJ) and 1\% lidocaine (5 mL IV; Elkins-Sinn, Inc) were given before sternotomy. Heparin was administered at the same dose every 30 minutes until the end of the experiment. A silk thread (0 silk, K834H; Ethicon, Inc, Somerville, NJ) was passed around the distal third of the left anterior descending artery (LAD), and both ends of the silk tie were threaded through a small vinyl tube to form a snare [4].

\section{Cardiopulmonary Bypass}

CPB was initiated at a flow rate of $75 \mathrm{~mL} / \mathrm{kg} / \mathrm{min}$, with a two-staged cannula for venous return and the right subclavian artery for arterial inflow. To maintain hematocrit levels, CPB pumps were primed with autologous blood drawn from a donor pig [4]. All animals were allowed to stabilize for 30 minutes after cannulation for CPB and then subjected to 30 minutes of normothermic regional ischemia by tightening the snare on the distal portion of the LAD and securing with a mosquito clamp [4]. Immediately after 30 minutes of regional ischemia, the ascending aorta was cross-clamped, and cold $\left(4^{\circ} \mathrm{C}\right)$ cardioplegia was administered antegrade through the aortic root using a cardioplegia needle (9F AR II aortic root cannula; Medtronics DLP, Inc., Grand Rapids, MI), inducing prompt diastolic arrest. DSA hearts $(n=6)$ received cold-blood cardioplegia $(10 \mathrm{~mL} / \mathrm{kg})$. DZX hearts $(n=6)$ initially received DSA cardioplegia $(10 \mathrm{~mL} / \mathrm{kg})$ containing diazoxide $(50 \mu \mathrm{mol} / \mathrm{L})$. Diazoxide was dissolved in dimethyl sulfoxide (DMSO; Fisher Scientific Co, Fair Lawn, NJ) before being added to DSA. DMSO was added as vehicle sham to DSA cardioplegia (final concentration less than $0.1 \%$ ). At 15 minutes of global ischemia, an additional $5 \mathrm{~mL} /$ $\mathrm{kg}$ of DSA cardioplegia without diazoxide was readministered in both groups. All hearts received topical hypothermia during global ischemia [4]. After 30 minutes of hypothermic global ischemia, the cross-clamp and the snare from the LAD were released, and the ice packing was removed and the hearts kept on total CPB for 120 minutes of reperfusion. Control hearts $(n=3)$ received CPB and sham reperfusion only.

\section{Tissue Samples}

Immediately after 120 minutes of reperfusion, myocardial tissue samples (approximately 15 $\times 30 \mathrm{~mm}$ each) from the area at risk (regional ischemic zone, six samples) and nonischemic area of left ventricle (global ischemic zone, six samples) consisting of epicardial, myocardial, and endocardial tissue were removed and divided into two samples. Regional ischemic and global ischemic zone samples were confirmed by monastryl blue pigment injection. The samples from each region were divided, equally and the subdivisions snap frozen, and used for either TUNEL (terminal deoxynucleotidyl transferase [TdT]-mediated dUTP nick end-labeling), protein isolation, or histology. One subdivided sample from each zone was routinely fixed for electron microscopy with Karnovsky fixative and embedded in epoxy resin [10].

\section{TUNEL-Mediated dUTP Nick End-Labeling}

TUNEL was performed using the ApopTag detection system (Intergen, Gaithersburg, MD) according to procedures described previously [10]. Thirty to 35 slides were selected for each sample. Five to six slides from each sample were assayed using both TUNEL and propidium iodide staining. Indirect digoxigenin fluorescence labeling of DNA was visualized as described previously [10]. Photomicrographs were taken in 10 to 15 random high-powered (20x) fields, and peroxidase TUNEL with methyl green counterstaining was performed on adjacent sequential serial slides ( $\mathrm{n}=5$ to 6 for each sample) to allow for morphologic evaluation of specimens. All cells were counted on each slide, and TUNEL-positive cells were expressed per 3,000 myocardial cells [10]. Myocardial cell specificity was determined on opposite adjacent sequential serial slides ( $\mathrm{n}=5$ to 6 for each sample) using the cardiacspecific monoclonal antibody for troponin I (Spectral Diagnostics Inc, Toronto, Ontario, 
Canada) labeled with antimouse IgG conjugated to Alexa 350 (Molecular Probes, Inc, Eugene, OR) [10]. Only those cells that could be confirmed by both peroxidase staining and Troponin I were classified as being of myocyte origin.

\section{Western Blot}

Myocardial proteins were isolated on iced SDS, Nonidet NP40 lysis buffer containing protease inhibitors ( $2 \mu \mathrm{mol} / \mathrm{L}$ complete; Boehringer Mannheim; Indianapolis, IN) and cellpermeable caspase inhibitors (DEVD.fmk, $10 \mu \mathrm{mol} / \mathrm{L}$, caspase-3-like irreversible inhibition; ZVAD.fmk, $50 \mu \mathrm{mol} / \mathrm{L}$, nonselective irreversible caspase inhibitor; Kamiya Biomedical Co, Seattle, WA). Protein content was determined using the Pierce BCA kit (Pierce Chemical Co, Rockford, IL). Equal amounts of protein $(50 \mu \mathrm{g})$ were fractionated on $10 \%$ SDSpolyacrylamide gels (Invitrogen, Casbad, CA) and then electroblotted to nitrocellulose (Invitrogen). Protein equivalency and transfer efficiency were confirmed by Ponceau red staining. Membranes were blocked for 2 hours at room temperature with 5\% nonfat milk in TBS $(25 \mathrm{mmol} / \mathrm{L}$ Tris, $137 \mathrm{mmol} / \mathrm{L} \mathrm{NaCl}$, and $2.7 \mathrm{mmol} / \mathrm{L} \mathrm{KCl})$ containing $0.05 \%$ Tween-20, and then incubated overnight with shaking at $4^{\circ} \mathrm{C}$ with specific antibodies according to methods previously described [11].

Immunoblotting was performed using monoclonal antiactin clone AC-40 (1:1000 dilution; Sigma; St. Louis, MO); bax (N-20) and bcl-2 (N-19) polyclonal antibodies (1:200 dilution each; Santa Cruz Biotechnology, Santa Cruz, CA); CCP-32 (Ab-3), anticaspase-3 monoclonal antibody (1:300 dilution; Oncogene, Boston, MA); and poly-ADP-ribose polymerase monoclonal antibody (PARP, Ab-2; Oncogene, Boston, MA). After washing, the blots were incubated for 1 hour with appropriate horseradish peroxidase-conjugated antibodies, and the blots detected using ECL Plus (Amersham Pharmacia Biotech, Piscataway, NJ).

\section{Semiquantitative Analysis}

Semiquantitative analysis was performed using an LKB Ultrascan XL laser densitometer (Pharmacia LKB). The integrals for each blot were calculated using the LKB GelScan XL software program for 1-D analysis [11].

\section{Caspase-3-Like Activity}

Protein samples for the estimation of caspase-3-like activity were isolated, separately, without caspase inhibitors. Caspase-3-like activity was estimated by colorimetric assay according to the procedures of the manufacturer (Chemicon International, Temecula, CA). Results were calibrated by standard curve using known concentrations of DEVD-pNA.

\section{Statistical Analysis}

The mean \pm SEM is shown for all variables. Statistical significance was determined by a mixed models analysis of variance (MM-ANOVA), which allows for both fixed and random effects and allows for more than one observation per animal (regional and global). In the analysis, when more than one observation was used from an animal, the site of ischemia (regional or global) was treated as a repeated measurement and the specific animal was incorporated as a random effect. We also tested whether different correlations for the repeated measurements in the DSA and DZX groups were necessary. All analyses were done in SAS(R) for Windows release 8.02 (SAS Institute, Cary, NC). Post hoc comparisons were made with the use of a Bonferroni correction. A $p$ less than 0.05 was considered statistically significant. 


\section{Results}

\section{Hemodynamics and Coronary Flow}

No significant differences in heart rate, left ventricular peak developed pressure, left ventricular end-diastolic pressure, systolic shortening, preload recruitable stroke work, tau, or the positive or negative change in pressure over time were observed between groups during equilibrium, after cannulation, during cardiopulmonary bypass, during regional ischemia or global ischemia, or during reperfusion [4]. Diazoxide had no significant effect on mean arterial pressure or coronary flow [4]. There were no experimental exclusions.

\section{TUNEL}

The area at risk was $13.91 \% \pm 1.17 \%$ left ventricular (LV) mass in DSA hearts and $14.11 \%$ $\pm 1.79 \% \mathrm{LV}$ mass in DZX hearts. No significant difference in area at risk was observed between groups. Apoptosis estimated by TUNEL-positive cells per 3,000 myocytes after 120 minutes of reperfusion was significantly increased $(p<0.05)$ in the zone of the heart made regionally ischemic by snaring of the distal portion of LAD (regional) as compared with the zone of the heart subjected only to global ischemia from aortic cross-clamping (Global) in both hearts receiving DSA cardioplegia (DSA) or DSA cardioplegia containing $50 \mu \mathrm{mol} / \mathrm{L}$ diazoxide (DZX). TUNEL-positive nuclei per 3,000 myocytes in the regional ischemic zones was $120.3 \pm 48.8$ in DSA and was significantly decreased $(p<0.05)$ to 21.4 \pm 5.3 in DZX hearts (Fig 1). Fewer than 20 TUNEL-positive nuclei per 3,000 myocytes were observed in global ischemic zones in DSA and DZX hearts (NS). There was no significant difference in TUNEL-positive nuclei per 3,000 myocytes between DZX regional and DSA or DZX global hearts.

\section{Caspase-3, Poly ADP-Ribose Polymerase (PARP), Bax, and Bcl-2}

The effects of DSA and DZX cardioplegia on caspase-3 and PARP latent zymogens (116 and $32 \mathrm{kDa}$, respectively) are shown in Figure 2. Results show the presence of catalytically active cleavage products of caspase-3 (20 kDa) and PARP ( $85 \mathrm{kDa}$ in the regional ischemic zones of both DSA and DZX. A significantly greater amount ( $p<0.05$ vs DZX) of catalytically active caspase-3 cleavage products was observed in DSA as compared with DZX hearts. No significant difference in PARP cleavage products $(85 \mathrm{kDa})$ was observed between DSA and DZX hearts in the regional ischemic zone. Caspase-3 and PARP cleavage products were undetectable in global ischemic zones and in control hearts. No difference in actin protein levels was observed between samples.

There was a slight nonsignificant increase of the anti-apoptotic protein bcl-2 in DZX as compared with control and DSA hearts in the regional ischemic zones (Fig 2). A significant increase $(p<0.05)$ in the levels of the pro-apoptotic protein bax was observed in both DSA and DZX hearts in the regional ischemic zones and in DSA hearts in the global zone. No increase in bax was observed between control hearts and DZX in the global ischemic zone.

Comparison of anti- to pro-apoptotic protein (bcl-2/bax) ratios (Fig 3) indicated a significant decrease $(p<0.05)$ in DSA hearts as compared with control and DZX hearts in the regional ischemic zones. No significant difference from control was observed in global zones between DSA and DZX hearts as compared with control hearts; however, there was a significant increase in bcl-2/bax ratio in DZX as compared with DSA hearts.

\section{Estimation of Catalytically Active Caspase-3-Like Activity}

A significant increase $(p<0.05$ vs control) in catalytically active caspase-3-like activity (pMols DVED-pNA $\mu \mathrm{g}$ protein/min) was observed in the regional ischemic zones of both DSA and DZX hearts as compared with control hearts; however, this increase was found to 
be significantly increased $(p<0.05)$ approximately threefold in DSA as compared with DZX hearts (Fig 4). Caspase activity was significantly increased $(p<0.05)$ in DSA hearts in the regional as compared with DSA and DZX hearts in the global ischemic zones. There was no significant difference in caspase activity between DZX in the regional ischemic zone and DSA and DZX hearts in the global ischemic zones. No significant difference was observed between DSA and DZX hearts in the global ischemic zones (NS vs control).

\section{Histology and Transmission Electron Microscopy}

Examination of myocardial tissue sections from regional and global ischemic zones from DSA and DZX hearts ( $\mathrm{n}=16$ to 18 slides from each group) indicated the consistent presence of severe tissue disruption with capillary dilatation in DSA hearts that was not evident in DZX hearts (Fig 5). There were no apparent differences in preexisting fibrosis between groups.

Transmission electron microscopy (Fig 6) indicated no apparent myocyte structural damage; however, the myofilaments in DSA hearts were found to be separated by numerous densely packed mitochondria with extensive swelling of the matrix and disruption of the cristae. The mitochondria in DSA hearts were filled with cristae, but the cristae was dilated. The intracristae matrix was electron lucent and there were prominent mitochondrial granules. In contrast, in DZX hearts, the mitochondria were packed with cristae, the intracristae matrix was electron dense, and whereas mitochondrial granules were observed, the quantity and size of these granules was significantly less than that observed in DSA hearts. Control hearts showed preserved myofilament structure with electron-dense intracristae matrix and few small mitochondrial granules.

\section{Wet Weight/Dry Weight Ratios}

Wet weight/dry weight ratios in DSA hearts were $5.8 \pm 0.6$ and $5.3 \pm 0.3$ for regional and global ischemic zones, respectively, and in DZX hearts were $5.9 \pm 0.4$ and $5.4 \pm 0.3$ for regional and global ischemic zones, respectively. Control heart wet weight/dry weight ratio was $5.4 \pm 0.3$. No significant difference in wet weight/dry weight ratio was observed within or between groups.

\section{Comment}

In this paper, we have investigated the mechanisms by which diazoxide provides for enhanced myocardial protection using a model of acute myocardial infarction [4]. In this model, a region of the left ventricle is made ischemic, cardioplegic arrest and crossclamping occurs, and then the snare and the cross-clamp are released after bypass, allowing for the investigation of regional ischemic injury similar to that observed in the patient under conditions that more closely approximate those experienced during surgery. This model mimics plaque rupture where a previously minimally obstructed vessel becomes acutely occluded without the formation and development of collateral coronary flow. The LAD snare was left in place throughout the 30 minutes of normothermic regional ischemia and the 30 minutes of hypothermic global arrest to ensure that no regional flow to the regional ischemic zone occurred and to mimic the effects of cardioplegia before distal anastomosis in a model in which no collateral coronary flow is present. This protocol resulted in the compartmentalization of the myocardium such that no cardioplegia was delivered to the regional ischemic zone of the left ventricular. It is only during reperfusion that coronary flow was reinstated and circulating diazoxide was perfused into this area.

Our results indicate that the addition of the selective mitoK $_{\mathrm{ATP}}$ channel opener, diazoxide ( $50 \mu \mathrm{mol} / \mathrm{L})$, to DSA cardioplegia significantly decreases apoptosis, by significantly 
decreasing caspase- 3 cleavage and caspase- 3 activity. We also show that anti-to-proapoptotic protein ratios are significantly decreased $(p<0.05)$ in DSA hearts as compared with control and DZX in the regional ischemic zones and are associated with mitochondrial structural damage.

We speculate that the decrease in apoptosis seen in our investigation is due to circulating diazoxide during reperfusion. Diazoxide is highly bound to albumin and has a plasma halflife of 22 hours [12]. These properties would allow diazoxide to provide for cardioprotection during the reperfusion phase of the regional ischemic zone. In the present report, we have used antegrade perfusion of cardioplegia and we speculate that retrograde perfusion may further limit infarct size and apoptosis.

In our investigations, $50 \mu \mathrm{mol} / \mathrm{L}$ diazoxide was added to DSA cardioplegia (600 mL) only during the initial administration of cardioplegia, and was not included when DSA was readministered after 15 minutes of global ischemia, based on preliminary results that showed that readministration of diazoxide resulted in a significant decrease in mean arterial pressure upon reperfusion. Diazoxide $(50 \mu \mathrm{mol})$ in the initial administration of cardioplegia followed by cardioplegia without diazoxide in all subsequent readministrations had no effect on heart rate, mean arterial pressure, or coronary flow as compared with DSA [4]. The circulating concentration of diazoxide was not measured in this study but based upon blood volumes of 3 to $4 \mathrm{~L}$ in the pig. A circulating diazoxide concentration of approximately 7.5 to $10 \mu \mathrm{mol}$ was present. This concentration of diazoxide and the effects seen in our study are in agreement with the investigations of Garlid and associates [13, 14], who have shown that diazoxide decreases cell injury in a dose-dependent manner at concentration between 1 and $30 \mu \mathrm{mol} / \mathrm{L}$.

Garlid and associates have suggested that the mechanism by which diazoxide modulates infarct size involves the regulation of mitochondrial volume and electron transport, and that these mechanisms are preeminent in maintaining mitochondrial function in the intact myocardium, allowing for enhanced preservation of adenine nucleotides during ischemia and efficient energy transfer during reperfusion [13-15]. It has also been suggested that mitochondrial membrane depolarization caused by $\mathrm{K}^{+}$entry through the opening of

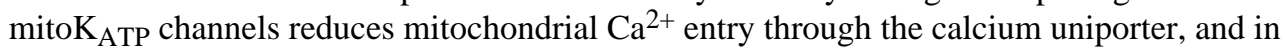
turn acts to reduce mitochondrial $\mathrm{Ca}^{2+}$ overload [16]. Subsequently, these events are believed to result in ATP production and cell salvage [17].

Support for these putative mechanisms comes from studies indicating that increased mitochondrial $\mathrm{Ca}^{2+}$ accumulation destabilizes the inner mitochondrial membrane, causing the inner membrane pores to open, which permits further movement of cations across the mitochondrial membrane [17]. The opening of these pores renders the mitochondrion incapable of synthesizing ATP, suggested to be a key event in the process leading to myocardial cell death. This would agree with our earlier reports, in which we suggested that modulation of mitochondrion "futile calcium cycling" may be of significance in the modulation of ischemic myocardial injury [3]. This hypothesis has been supported by Pacher and Hajnoczky [18], who have shown that mitochondrial $\mathrm{Ca}^{2+}$ uptake is crucial for the initiation of apoptosis but can be modulated by antiapoptotic proteins (bcl-2).

The expression of bcl-2-related proteins has been shown to be a key element in the genesis of apoptosis and is known to affect mitochondrial function and regulate the release of apoptosis-activating factors [19]. Recent investigations have shown that ATP depletion in cells initiates the translocation of bax, a pro-apoptotic bcl-2 family member protein, from the cytosol to the outer mitochondrial membrane, by mechanisms that remain unclear [20]. The translocation of bax is thought to cause mitochondrial dysfunction and swelling, and to 
induce the efflux of cytochrome $\mathrm{c}$ to the cytosol by a mechanism that is thought to involve changes in mitochondrial permeability caused by the opening of the permeability transition pore in the mitochondrial inner membrane, or mitochondrial hyperpolarization followed by swelling and membrane rupture [21]. Cytochrome c, on release to the cytosol, activates caspases, normally expressed as latent zymogens, which are cleaved and reassociated to generate catalytically active heterodimers.

Bcl-2 is an antiapoptotic protein that inhibits the oligomerization of bax and prevents apoptosis by preserving mitochondrial integrity and suppressing cytochrome $\mathrm{c}$ release without ameliorating ATP depletion [22]. It is the activation of the effector caspase, caspase-3, responsible for the cleavage of poly ADP-ribose polymerase (PARP), a nuclear enzyme that is catalytically activated by DNA strand interruptions, that is the common event initiated by the multiple different stimuli that induce apoptosis [22].

Our results show that anti-to-pro-apoptotic protein (bcl-2/bax) ratios are significantly decreased $(p<0.05)$ in DSA hearts as compared with control and DZX hearts in the regional ischemic zones, and that there was a significant increase in the anti-to-pro-apoptotic protein ratio in DZX as compared with DSA hearts in the global ischemic zone. We further show that diazoxide preserved mitochondrial structure. These results are in agreement with the proposed mechanism of apoptotic signal transduction and mitochondrial structure in the apoptotic cascade [19-22].

Recently, Ozcan and associates [23] have suggested that treatment of mitochondria with diazoxide during the anoxic period effectively preserves the structural and functional integrity of mitochondria, maintaining ATP generation after the anoxic insult. This would agree with our observations using transmission electron microscopy, which show extensive swelling of the mitochondrial matrix and disruption of cristae in the regional ischemic zone of DSA hearts, which is less pronounced in the global ischemic zone in DSA hearts. Only a slight swelling of the intercristae matrix was observed in either the regional or global ischemic zones in DZX hearts.

We also observed large electron-opaque mitochondrial granules in DSA hearts. These granules are also present in DZX hearts, but are significantly smaller in size and significantly less numerous than those observed in DSA hearts. Previous investigations have shown that these granules contain calcium and inorganic phosphate [24]. When modest amounts of calcium ions are present, they are deposited in these granules; however, if a large increase in calcium occurs de novo, granule formation occurs [24]. The increased size and number of granules seen in DSA hearts as compared with DZX hearts would suggest that there is an increased accumulation of mitochondrial $\mathrm{Ca}^{2+}$ in DSA hearts that is attenuated in DZX hearts. This would agree with the recent observations of Murata and associates [25], who have shown that opening mitoK $\mathrm{ATP}_{\mathrm{A}}$ and partial mitochondrial membrane depolarization attenuates mitochondrial $\mathrm{Ca}^{2+}$ overload.

Whereas the mechanism(s) by which diazoxide effects infarct limitation remains to be elucidated, our results indicate that at least in part, the addition of diazoxide to DSA cardioplegia significantly decreases apoptosis through the modulation of bcl-2/bax ratio and by decreasing caspase- 3 activity and preserving mitochondrial structure in the in situ bloodperfused pig heart model of acute myocardial infarction, and represents an additional modality for enhancing myocardial protection.

\section{Acknowledgments}

This study was supported by the National Institutes of Health (HL-29077, HL-59542). 


\section{References}

1. Tsukube T, McCully JD, Federman M, Krukenkamp IB, Levitsky S. Developmental differences in cytosolic calcium accumulation associated with surgically induced global ischemia: optimization of cardioplegic protection and mechanism of action. J Thorac Cardiovasc Surg. 1996; 112:175-84. [PubMed: 8691865]

2. Tsukube T, McCully JD, Metz RM, Cook U, Levitsky S. Amelioration of ischemic calcium overload correlates with high energy phosphates in the senescent myocardium. Am J Physiol (Heart Circ Physiol). 1997; 42:H418-27.

3. Faulk EA, McCully JD, Tsukube T, Hadlow NC, Krukenkamp IB, Levitsky S. Myocardial mitochondrial calcium accumulation modulates nuclear calcium accumulation and DNA fragmentation. Ann Thorac Surg. 1995; 60:338-44. [PubMed: 7544101]

4. Wakiyama H, Cowan DB, Toyoda Y, Federman M, Levitsky S, McCully JD. Selective opening of mitochondrial ATP-sensitive potassium channels during cardiopulmonary bypass decreases apoptosis and necrosis in a model of acute myocardial infarction. Eur J Cardiothorac Surg. 2002; 21:424-33. [PubMed: 11888758]

5. Toyoda Y, Levitsky S, McCully JD. Opening of mitochondrial ATP-sensitive potassium channels enhances cardioplegic protection. Ann Thorac Surg. 2001; 71:1281-9. [PubMed: 11308175]

6. Ducko CT, Stephenson ER Jr, Jayawant AM, Vigilance DW, Damiano RJ Jr. Potassium channel openers: are they effective as pretreatment or additives to cardioplegia? Ann Thorac Surg. 2000; 69:1363-8. [PubMed: 10881806]

7. Maskal SL, Cohen NM, Hsia PW, Wechsler AS, Damiano RJ Jr. Hyperpolarized cardiac arrest with a potassium-channel opener, aprikalim. J Thorac Cardiovasc Surg. 1995; 110:1083-95. [PubMed: 7475137]

8. Monti F, Iwashiro K, Picard S, et al. Adenosine triphosphate-dependent potassium channel modulation and cardioplegia-induced protection of human atrial muscle in an in vitro model of myocardial stunning. J Thorac Cardiovasc Surg. 2000; 119:842-8. [PubMed: 10733778]

9. Buja LM, Entman ML. Modes of myocardial cell injury and cell death in ischemic heart disease. Circulation. 1998; 98:1355-7. [PubMed: 9760287]

10. Stadler B, Phillips J, Toyoda Y, Federman M, Levitsky S, McCully JD. Adenosine enhanced ischemic preconditioning modulates necrosis and apoptosis: effects of stunning and ischemia/ reperfusion. Ann Thorac Surg. 2001; 72:555-63. [PubMed: 11515897]

11. McCully JD, Lotz MM, Krukenkamp IB, Levitsky S. A brief period of retrograde hyperthermic perfusion enhances myocardial protection from global ischemia: association with accumulation of Hsp 70 mRNA and protein. J Mol Cell Cardiol. 1996; 28:231-41. [PubMed: 8729056]

12. Sellers EM, Koch-Weser J. Binding of diazoxide and other benzothiadiazines to human albumin. Biochem Pharmacol. 1974; 23:553-66. [PubMed: 4822742]

13. Garlid KD, Paucek P, Yarov-Yarovoy V, et al. The mitochondrial $\mathrm{K}_{\mathrm{ATP}}$ channel as a receptor for potassium channel openers. J Biol Chem. 1996; 271:8796-9. [PubMed: 8621517]

14. Garlid K, Paucek P, Yarov-Yarovoy V, et al. Cardioprotective effect of diazoxide and its interaction with mitochondrial ATP-sensitive $\mathrm{K}^{+}$channels: possible mechanism of cardioprotection. Circ Res. 1997; 81:1072-82. [PubMed: 9400389]

15. Dos Santos P, Kowaltowski AJ, Laclau MN, et al. Mechanisms by which opening the mitochondrial ATP-sensitive $\mathrm{K}(+)$ channel protects the ischemic heart. Am J Physiol Heart Circ Physiol. 2002; 283:H284-95. [PubMed: 12063301]

16. Holmuhamedov EL, Jovanovic S, Dzeja PP, Jovanovic A, Terzic A. Mitochondrial ATP-sensitive $\mathrm{K}+$ channels modulate cardiac mitochondrial function. Am J Physiol (Heart Circ Physiol). 1998; 275:H1567-6.

17. Ferrari R. The role of mitochondria in ischemic heart disease. J Cardiovasc Pharmacol. 1996; 28(Suppl):S1-10. [PubMed: 8891865]

18. Pacher P, Hajnoczky G. Propagation of the apoptotic signal by mitochondrial waves. EMBO J. 2001; 20:4107-21. [PubMed: 11483514]

19. Kroemer G, Reed JC. Mitochondrial control of cell death. Nature Med. 2000; 6:513-9. [PubMed: 10802706] 
20. Vaux DL, Strasser A. The molecular biology of apoptosis. Proc Natl Acad Sci USA. 1996; 93:2239-44. [PubMed: 8637856]

21. Liu X, Kim CN, Yang J, Jemmerson R, Wang X. Induction of apoptotic program in cell-free extracts: requirement for dATP and cytochrome c. Cell. 1996; 86:147-57. [PubMed: 8689682]

22. Sakahira H, Enari M, Nagata S. Cleavage of CAD inhibitor in CAD activation and DNA degradation during apoptosis. Nature. 1998; 391:96-9. [PubMed: 9422513]

23. Ozcan C, Holmuhamedov EL, Jahangir A, Terzic A. Diazoxide protects mitochondria from anoxic injury: implication for myopreservation. J Thorac Cardiovasc Surg. 2001; 121:298-306. [PubMed: 11174735]

24. Ghadially, FN., 3rd, editor. Ultrastructural Pathology of the cell and matrix. Vol. vol 1. Butterworths; Boston: 1988. p. 232-8.

25. Murata M, Akao M, O’Rourke B, Marbán E. Mitochondrial ATP-sensitive potassium channels attenuate matrix $\mathrm{Ca}^{2+}$ overload during simulated ischemia, and reperfusion: possible mechanism of cardioprotection. Circ Res. 2001; 89:891-8. [PubMed: 11701616] 


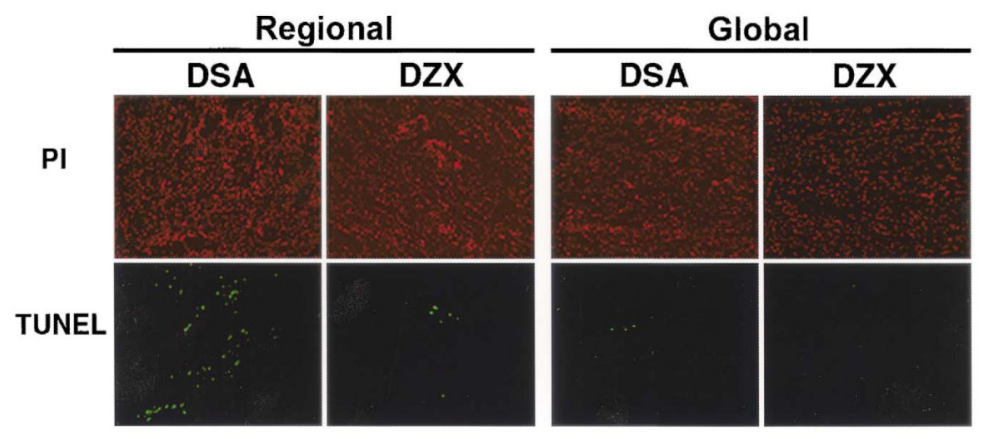

Fig 1.

TUNEL-positive nuclei/3,000 myocytes in the regional and global ischemic zones. Representative photomicrographs of TUNEL photomicrographs of regional ischemia (Regional) and global ischemia (Global) sections $(6 \mu \mathrm{m})$ from hearts receiving Deaconess Surgical Associates (DSA) cardioplegia or DSA cardioplegia containing $50 \mu \mathrm{mol}$ diazoxide (DZX) after 120 minutes of reperfusion. Control hearts received cardiopulmonary bypass and sham reperfusion only and are shown for comparative purposes. Myocyte nuclei were visualized by indirect digoxigenin fluorescence labeling of DNA with propidium iodide (PI), shown as red in top row. TUNEL-positive cells were visualized by FITC labeling and are shown as green in bottom row. Results indicate a significant increase in TUNEL-positive nuclei/3,000 myocytes in DSA regional as compared with DZX regional and DSA and DZX global. 

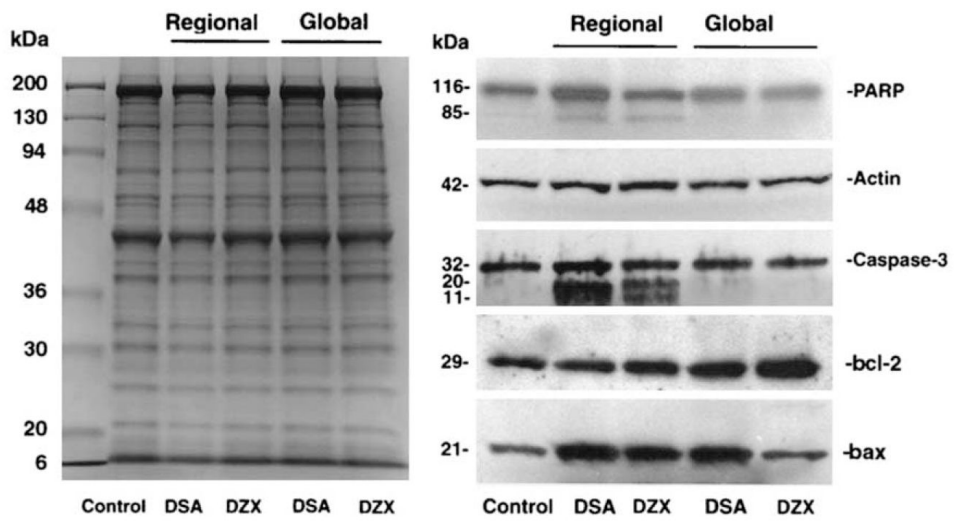

Fig 2.

The effects of Deacon Surgical Associates (DSA) and diazoxide (DZX) cardioplegia on poly $A D P$-ribose polymerase (PARP) and caspase-3 cleavage and antiapoptotic bcl-2 and proapoptotic bax levels determined by Western blot in the regional and global ischemic zones. Protein equivalency and transfer efficiency were confirmed by Ponceau red staining and actin immunoblotting. A representative $10 \%$ polyacrylamide gel stained with Coomassie brilliant blue R-250 is shown on the left. Molecular mass (kDa) is shown. Representative immunoblots for PARP, actin, caspase-3, bax, and bcl-2 are shown on the right. Latent zymogens for PARP and caspase- 3 are shown at 116 and $32 \mathrm{kDa}$, respectively. Catalytically active proteases for PARP and caspase- 3 are shown at $85 \mathrm{kDa}$ for PARP and 20 and $11 \mathrm{kDa}$ for caspase-3. Protein equivalency and transfer efficiency were confirmed by Ponceau red staining and actin immunoblotting. Results indicate a significant increase in caspase-3 cleavage in DSA regional as compared with DZX regional and DSA and DZX global. 


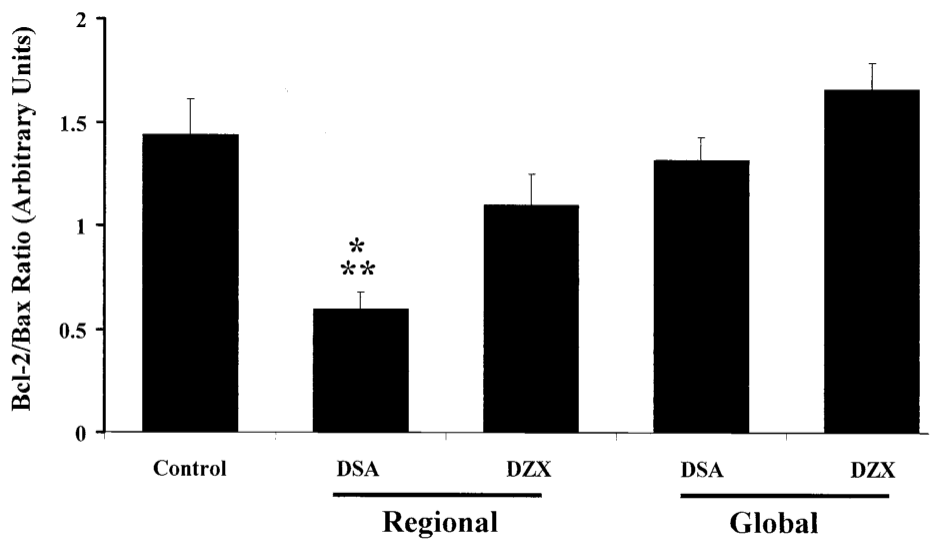

Fig 3.

The effects of Deaconess Surgical Associates (DSA) and diazoxide (DZX) cardioplegia on bcl-2/bax ratio (arbitrary units) in the regional and global ischemic zones. All results are shown as mean \pm SEM for $n=6$ for each sample. Significant differences at $\mathrm{p}$ less than 0.05 are shown as *versus control and as **versus DZX. Results indicate a significant decrease in anti- to pro-apoptotic protein levels in DSA regional as compared with DZX regional and DSA and DZX global ischemic zones of hearts. 


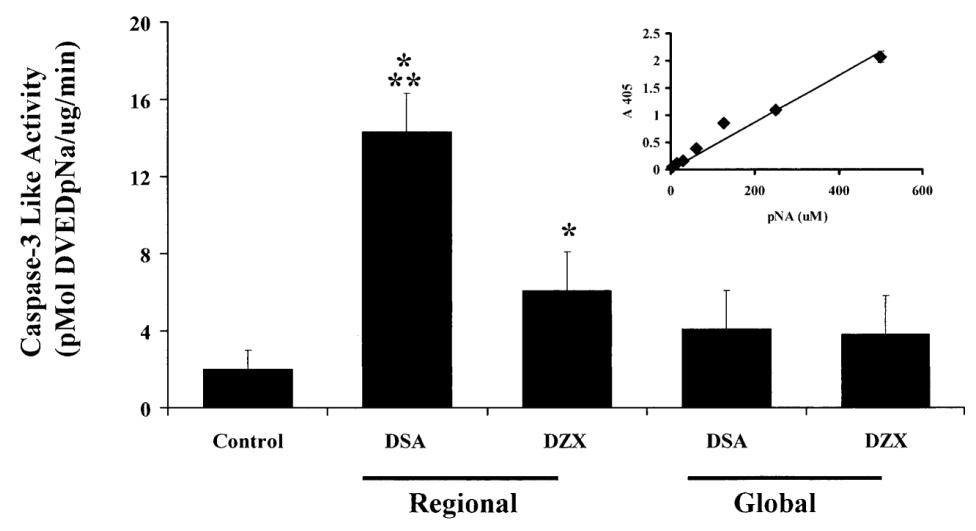

Fig 4.

The effects of Deaconess Surgical Associates (DSA) and diazoxide (DZX) cardioplegia on catalytically active caspase $3-$ like activity (pmol DVED-pNA/ $\mu \mathrm{g}$ protein/min) in the regional and global ischemic zones. Catalytically active caspase-3-like activity (pmol DVED-pNA/ $\mu \mathrm{g}$ protein/min) was significantly increased $(\mathrm{p}<0.05$ ) approximately threefold in DSA as compared with DZX hearts in the regional ischemic zone. Standard curve using known concentrations of DVED-pNA is shown in inset. All results are shown as mean \pm SEM for $\mathrm{n}=6$ for each sample. Significant differences at $\mathrm{p}$ less than 0.05 are shown as *versus control and as **versus DZX. Results indicate a significant increase in caspase-3like activity in DSA regional as compared with DZX re gional and DSA and DZX global. 

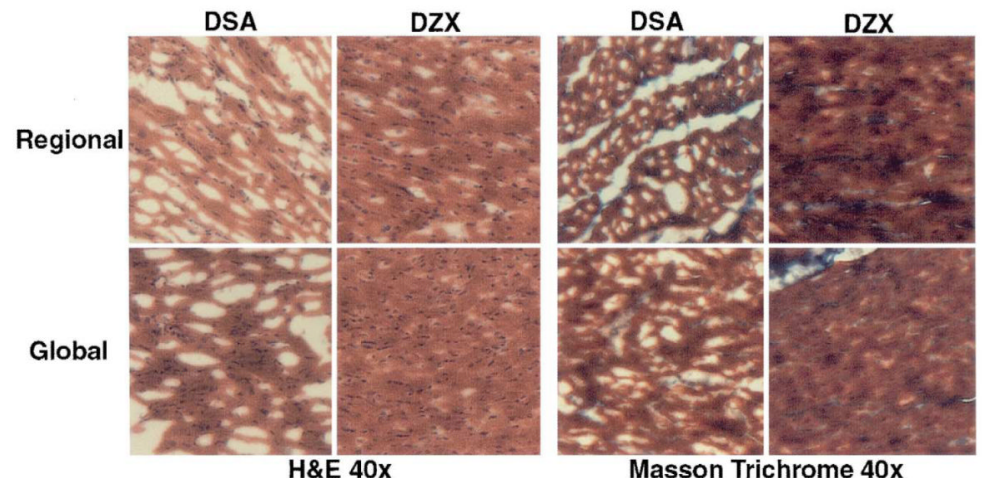

Fig 5.

Representative hematoxylin and eosin $(\mathrm{H} \& \mathrm{E}, \times 40)$ and Masson trichrome $(\times 40)$ stained myocardial sections $(6 \mu \mathrm{m})$ from regional and global ischemic zones of Deaconess Surgical Associates (DSA) and diazoxide (DZX) hearts. Results indicate severe tissue disruption with capillary dilatation in DSA hearts that was not evident in DZX hearts. 

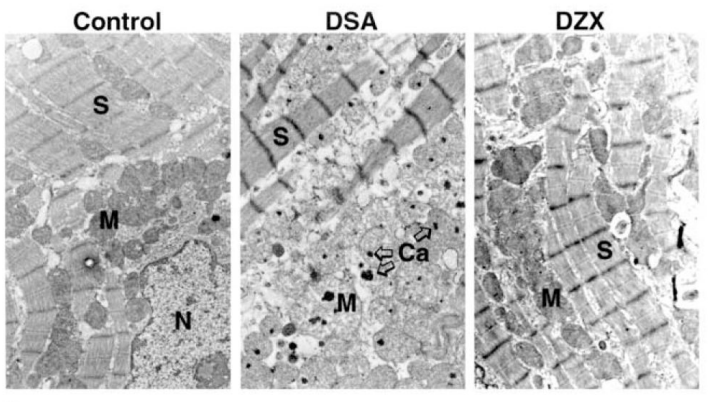

A
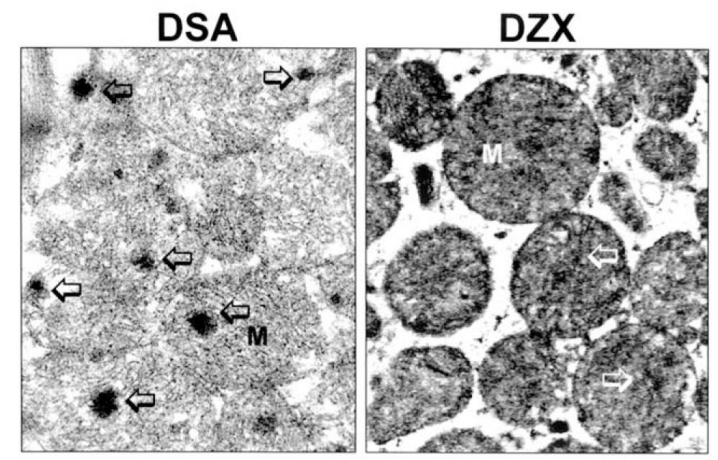

B

Fig 6.

(A) Representative transmission electron micrographs $(\times 10,500)$ from regional ischemic zones from control, Deaconess Surgical Associates (DSA), and diazoxide (DZX) hearts. Results show myocytes in DSA hearts are separated by numerous densely packed, dilated mitochondria with extensive swelling of the matrix and disruption of the cristae and numerous large mitochondrial granules. Control and DZX hearts show preserved myofilament structure with electron-dense intracristae matrix and few small mitochondrial granules. $(\mathrm{Ca}=$ calcium granules $; \mathrm{M}=$ mitochondrion; $\mathrm{N}=$ nucleus $; \mathrm{S}=$ sarcomere $)(\mathrm{B})$

Representative transmission electron micrographs $(\times 62,500)$ of mitochondria from regional ischemic zones from DSA and DZX hearts. Results show disrupted mitochondrial structure and large mitochondrial granules in DSA hearts. DZX hearts show preserved mitochondrial structure with smaller significantly less mitochondrial granules as compared with DSA hearts. Mitochondrial granules are indicated by arrows. 